\title{
Temporal differences in culturable severe acute respiratory coronavirus virus 2 (SARS-CoV-2) from the respiratory and gastrointestinal tracts in a patient with moderate coronavirus disease 2019 (COVID-19)
}

\author{
Jennifer M. Audsley PhD ${ }^{1, a}$ (1), Natasha E. Holmes MBBS, PhD ${ }^{2,3,4, a}$, Francesca L. Mordant MSc ${ }^{5}$, Celia Douros BSc ${ }^{6}$, Sara \\ E. Zufan $\mathrm{MPH}^{7}$, Thi H. O. Nguyen $\mathrm{PhD}^{5}$, Lukasz Kedzierski $\mathrm{PhD}^{5}$, Louise C. Rowntree $\mathrm{PhD}^{5}$, Luca Hensen $\mathrm{MSc}^{5}$, \\ Kanta Subbarao MBBS, MPH ${ }^{5,8}$, Katherine Kedzierska $\mathrm{PhD}^{5}$, Suellen Nicholson BSci6 ${ }^{6}$, Norelle Sherry MBBS \\ Irani Thevarajan MBBS, $\mathrm{PhD}^{1,9}$, Thomas Tran BAppSci ${ }^{6, \mathrm{~b}}$ and Julian Druce $\mathrm{PhD}^{6, \mathrm{~b}}$ \\ ${ }^{1}$ Department of Infectious Diseases, The University of Melbourne at the Peter Doherty Institute for Infection and Immunity, Melbourne, Australia, ${ }^{2}$ Department of \\ Infectious Diseases, Austin Hospital, Heidelberg, Australia, ${ }^{3}$ Department of Medicine and Radiology, The University of Melbourne, Australia, ${ }^{4}$ Data Analytics \\ Research and Evaluation (DARE) Centre, Austin Health and The University of Melbourne, Heidelberg, Australia, ${ }^{5}$ Department of Microbiology and Immunology, \\ University of Melbourne at The Peter Doherty Institute for Infection and Immunity, Melbourne, Australia, ${ }^{6}$ Victorian Infectious Diseases Reference Laboratory, \\ The Royal Melbourne Hospital at The Peter Doherty Institute for Infection and Immunity, Melbourne, Australia, ${ }^{7}$ Microbiological Diagnostic Unit Public Health \\ Laboratory, Department of Microbiology and Immunology, The University of Melbourne at The Peter Doherty Institute for Infection and Immunity, Melbourne, \\ Australia, ${ }^{8}$ World Health Organisation (WHO) Collaborating Centre for Reference and Research on Influenza, at The Peter Doherty Institute for Infection and \\ Immunity, Melbourne, Australia and ${ }^{9}$ Victorian Infectious Diseases Services, The Royal Melbourne Hospital at the Peter Doherty Institute for Infection and \\ Immunity, Melbourne, Australia
}

To the Editor-Although infectivity from respiratory transmission of severe acute respiratory coronavirus virus 2 (SARS-CoV-2) is a well-recognized and evolving area, the role of fecal-oral transmission remains unclear and is a key area requiring further evaluation. SARS-CoV-2 shedding in the gastrointestinal tract (GIT) occurs later than in the respiratory tract. ${ }^{1}$ Here, we report the first case of virus isolation from feces later than respiratory samples and the value of exploring the role of fecal-oral transmission as an underappreciated contributor to infection transmission.

On returning from international travel in March 2020, a 63year-old man with well-controlled HIV presented to a tertiary-care hospital in Melbourne, Australia, and was subsequently diagnosed with moderate coronavirus disease 2019 (COVID-19). The patient was enrolled through the Sentinel Travelers Research Preparedness Platform for Emerging Infectious Diseases (SETREP-ID, ethics approvals HREC/17/MH/53 and UoM1749349) and provided written informed consent. His CD4 cell count was 973 cells/ $\mathrm{mm}^{3}$, and he had had an undetectable HIV viral load ( $<20$ cop$\mathrm{ies} / \mathrm{mL}$ ) for $>5$ years. He had a history of ischemic heart disease, stage 3 a chronic kidney disease, and previous non-Hodgkin's lymphoma treated with chemotherapy in 2012.

On admission (4 days after symptom onset), symptoms included diarrhea, history of fever (afebrile on admission), cough with sputum production, chest pain with coughing, myalgia, dyspnea, sore throat, and leg cramps. His oxygen saturation was $99 \%$ on room air and his respiratory rate was 30 breaths per minute. The

Author for correspondence: Dr Julian Druce, E-mail: julian.druce@mh.org.au. Or Dr Jennifer Audsley, E-mail: jennifer.audsley@unimelb.edu.au

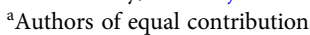

${ }^{\mathrm{b}}$ Authors of equal contribution.

Cite this article: Audsley JM, et al. (2022). Temporal differences in culturable severe acute respiratory coronavirus virus 2 (SARS-CoV-2) from the respiratory and gastrointestinal tracts in a patient with moderate coronavirus disease 2019 (COVID19). Infection Control \& Hospital Epidemiology, 43: 1286-1288, https://doi.org/10.1017/ ice. 2021.223 following day his chest pain and diarrhea resolved, but cough and sputum production were ongoing at hospital discharge 5 days later and the following 2 months. He did not receive any specific antiviral or corticosteroid treatment during admission according to the standard of care at the time.

SARS-CoV-2 viral RNA was detected using real-time reverse transcription polymerase chain reaction (RT-PCR) on days 5 and 9 in sputum and on days 6 and 9 in feces. PCR-positive samples were cultured to assess viral infectivity (Supplementary Methods online). Virus was successfully isolated from sputum only on day 5 , whereas virus was isolated from feces only on day 9. The cycle threshold $(\mathrm{Ct})$ values for sputum and feces from which virus was isolated were 25 and 23, respectively. Virus isolation was verified from culture medium by a RT-PCR Ct value of 15 . By day 53, all samples were RT-PCR negative. At the most recent follow-up (day 116), the patient had made a complete recovery and all samples remained RT-PCR negative (Fig. 1).

Neutralizing antibody was measured using 2 methods. The microneutralization assay revealed a rise in titer from undetectable to 254 on paired serum samples (days 5 and 53). The surrogate virus neutralization tests were performed in parallel on samples from days 5, 53, and 116. There was evidence of development of neutralizing antibody by day 53 , and persistence of antibodies at day 116 (Fig. 1). IgA, IgM, and IgG responses were positive by day 53 but only IgG persisted at a high level at day 116. In samples from this HIV-positive patient, broad immune activation was observed for both innate and adaptive immune cell subsets at day 5 compared to day 53 .

Single-nucleotide polymorphisms (SNPs) were not detected between all samples except the sputum sample from day 9 , which had 5 SNPs compared to the other samples. This sample had lower sequence quality (more heterozygous sites/lower genome coverage); thus, whether the genetic differences reflect RNA degradation, sequencing artifacts, or intrahost variation remains unclear. 


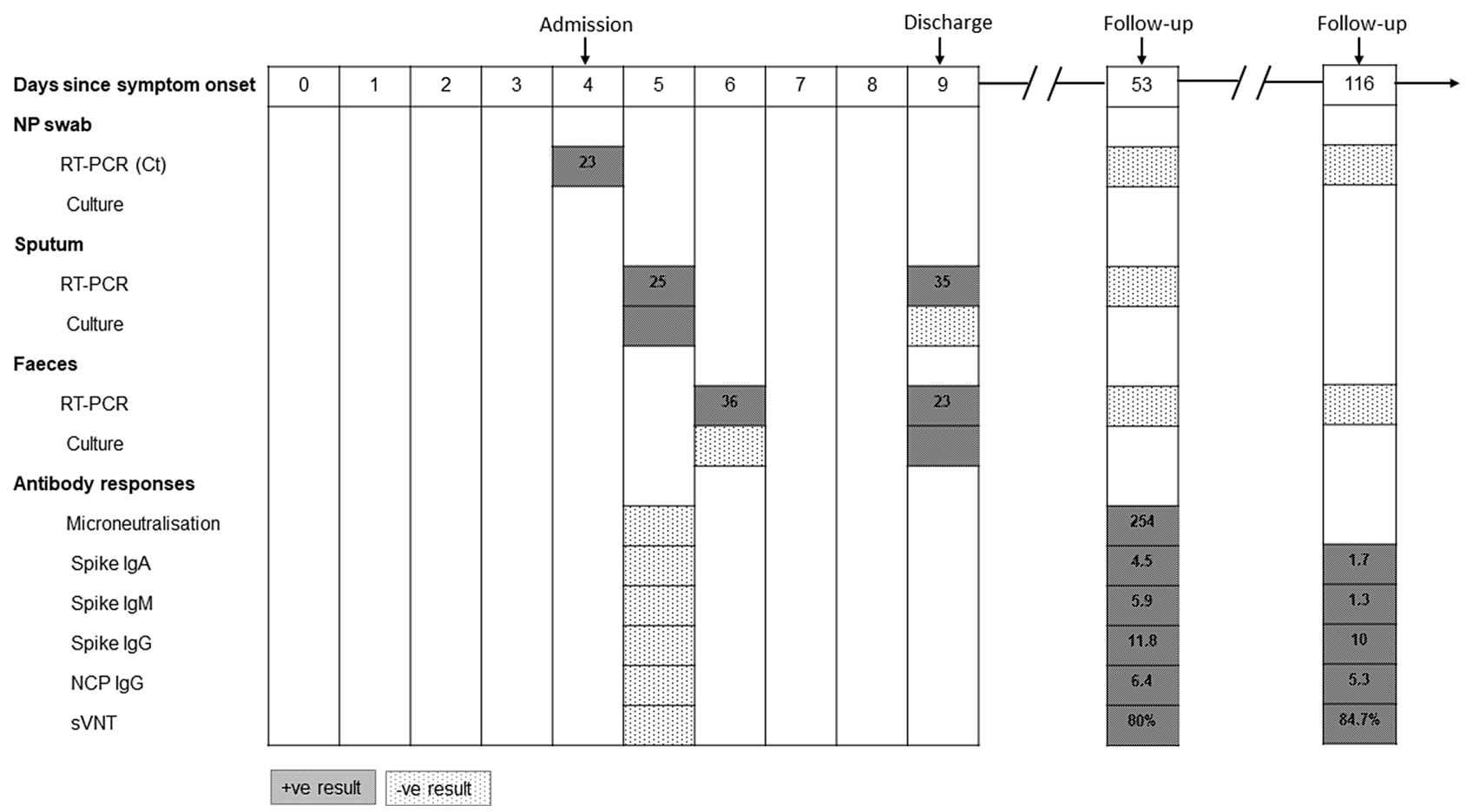

Fig. 1. SARS-CoV-2 shedding, viral isolation, and antibody development in a recovered HIV-positive COVID-19 patient. Red rectangles indicate positive results and green rectangles indicate negative or undetected results. The numbers in RT-PCR rectangles are cycle threshold (Ct) values. Numbers in antibody response rectangles are titers for microneutralization assays and index values for IgA/lgM/lgG. Note. Ig, immunoglobulin; NCP, nucleoscapsid; NP, nasopharyngeal; sVNT, surrogate virus neutralization test.

Understanding the drivers of SARS-CoV-2 transmission is critical to the pandemic response and for implementing key public health measures. The role of fecal-oral transmission is unclear but has been increasingly indicated with the isolation of infectious SARS-CoV-2 in fecal samples. ${ }^{2}$ Additionally, generation of possible fecal bioaerosols can lead to outbreaks in high-rise buildings ${ }^{3}$ and hospital settings. ${ }^{4}$ Although studies have shown that SARS-CoV-2 shedding in the GIT occurs later than in the respiratory tract, ${ }^{1}$ this is the first case that demonstrated culturable virus isolation from feces at a later time than respiratory samples.

Although this patient had well-controlled HIV, the link between an altered immune system and viable SARS-CoV-2 in the GIT is unclear. Altered viral transmission dynamics has been observed with influenza and immunosuppression unrelated to HIV infection. ${ }^{5,6}$

Importantly, the temporal difference of culturable virus from the respiratory tract and the GIT did not translate to genetic mutations between SARS-CoV-2 virus from the 2 anatomical sites, demonstrating that intrahost variation is unlikely, except for a single lower-quality sequence.

The prevalence of GIT symptoms in SARS-CoV-2 is $12 \%$, and viral RNA shedding has been identified in $41 \%$ of stool specimens. ${ }^{7}$ Angiotensin converting enzyme 2 (ACE-2) is highly expressed in the small intestine, enabling SARS-CoV-2 infection of the digestive system. SARS-CoV-2 has been detected in the intestinal biopsies from asymptomatic individuals 3 months after COVID- 19 onset. $^{8}$ The focus of infection control and public health measures are on preventing respiratory transmission, and diagnostics used to document viral clearance from the respiratory tract. This case demonstrates that after resolution of diarrhea and most acute symptoms, virus could be cultured from feces. By day 53 of disease onset, feces returned to RT-PCR negative, and although the exact timing is unknown, prolonged respiratory shedding to 60 days after disease onset has been observed. ${ }^{9}$

In summary, we report a case of virus isolation in feces when there was clinical improvement, and viable virus was not detectable in respiratory specimens. Fecal-oral transmission warrants further evaluation, including subgroups such as immunocompromised hosts. Gaining a better understanding of coronavirus disease 2019 (COVID-19) transmission dynamics is critical to this pandemic.

Acknowledgments. We sincerely thank the patient for his ongoing participation in SETREP-ID and for this case report.

Financial support. This work was supported by funding through the National Health and Medical Research Council Centre of Research Excellence (NHMRC CRE), the Australian Partnership for Preparedness Research on Infectious Disease Emergencies (APPRISE AppID 1116530) and in part through funding from the Jack Ma Foundation and the A2 Milk Company. K.S. is supported by an NHMRC Investigator grant. The Melbourne WHO Collaborating Centre for Reference and Research on Influenza is supported by the Australian Government Department of Health. L.H. was supported by Melbourne International Research Scholarships and Melbourne International Fee Remission Scholarships from The University of Melbourne.

Conflicts of interest. All authors have no conflicts relevant to this article.

Supplementary material. To view supplementary material for this article, please visit https://doi.org/10.1017/ice.2021.223

\section{References}

1. Wu Y, Guo C, Tang L, et al. Prolonged presence of SARS-CoV-2 viral RNA in faecal samples. Lancet Gastroent Hepatol 2020;5:434-435. 
2. Xiao F, Sun J, Xu Y, et al. Infectious SARS-CoV-2 in feces of patient with severe COVID-19. Emerg Infect Dis 2020;26:1920-1922.

3. Kang M, Wei J, Yuan J, et al. Probable evidence of fecal aerosol transmission of SARS-CoV-2 in a high-rise building. Ann Intern Med 2020;173: 974-980.

4. McDermott CV, Alicic RZ, Harden N, Cox EJ, Scanlan JM. Put a lid on it: are faecal bio-aerosols a route of transmission for SARS-CoV-2? J Hosp Infect 2020;105:397-398.

5. Klimov AI, Rocha E, Hayden FG, Shult PA, Roumillat LF, Cox NJ. Prolonged shedding of amantadine-resistant influenzae A viruses by immunodeficient patients: detection by polymerase chain reaction-restriction analysis. J Infect Dis 1995;172:1352-1355.
6. Weinstock DM, Gubareva LV, Zuccotti G. Prolonged shedding of multidrugresistant influenza A virus in an immunocompromised patient. N Engl J Med 2003;348:867-868.

7. Parasa S, Desai M, Thoguluva Chandrasekar V, et al. Prevalence of gastrointestinal symptoms and fecal viral shedding in patients with coronavirus disease 2019: a systematic review and meta-analysis. JAMA Netw Open 2020;3: e2011335.

8. Gaebler C, Wang Z, Lorenzi JCC, et al. Evolution of antibody immunity to SARS-CoV-2. bioRxiv 2020. doi: 10.1101/2020.11.03.367391.

9. Li J, Zhang L, Liu B, Song D. Case report: viral shedding for 60 days in a woman with COVID-19. Am J Trop Med Hygiene 2020;102:1210-1213.

\title{
Recurrence of coronavirus disease 2019 (COVID-19), future paths and challenges
}

\author{
Gabriel Savogin Andraus ${ }^{1}$ (1) , Viviane Maria de Carvalho Hessel Dias MD, MSc ${ }^{1,2}$ (1) and \\ Cristina Pellegrino Baena MSc, $\mathrm{PhD}^{1}$ (1) \\ ${ }^{1}$ Pontifícia Universidade Católica do Paraná, Escola de Medicina, Curitiba, Paraná, Brazil and ${ }^{2}$ Hospital Marcelino Champagnat, Curitiba, Paraná, Brazil
}

To the Editor - Currently, coronavirus disease 2019 (COVID-19) recurrence is a poorly understood phenomenon concerning recently initiated vaccination efforts, novel genotypic variants of SARS-CoV-2, and population-level transmission dynamics. To comprehend the true impact of recurrence for both the patient and population, it is imperative that different possible mechanisms of recurrence are identified and appropriately investigated.

In this regard, we propose that symptom chronology, serial reverse transcription-quantitative polymerase chain reaction (RT-qPCR) tests, viral culture, and viral genome sequencing are important components in the investigation of suspected COVID-19 recurrence cases. These techniques allow differentiation between recurrence ${ }^{1-5}$ and persistence ${ }^{6}$ and estimation of viral load. They are useful for determining viral viability for infectiousness ${ }^{7}$ and for confirming reinfection via the detection of distinct genetic variants of SARS-CoV-2. ${ }^{5,8}$ Together, these variables confer sufficient data for classifying cases according to relevant clinical and laboratory parameters to guide decision making on both patient and population levels. Such enhanced decision making may be essential considering the impact of the novel SARS-CoV-2 variants of concern (B.1.1.7, B.1.351, P.1, B.1.427 and B.1.429) on patient outcomes and infectiousness. ${ }^{8}$

In COVID-19 recurrence, recurring positive patients present with a new positive RT-qPCR test after the apparent resolution of the initial SARS-CoV-2 infection. ${ }^{1-3}$ Although purely a laboratory classification, positive recurrence is a relatively common phenomenon, with $9 \%-16 \%$ of hospitalized patients being susceptible to positive recurrence in an RT-qPCR test. ${ }^{1-3}$ It is important to distinguish patients with RT-qPCR recurrence from those with clinically significant symptom relapse, defined as an intermission in symptoms marked by apparent clinical cure. ${ }^{4}$ Symptom

Author for correspondence: Viviane Maria de Carvalho Hessel Dias, E-mail: carvalhohdias@gmail.com

Cite this article: Andraus GS, Dias VMdCH, and Baena CP. (2022). Recurrence of coronavirus disease 2019 (COVID-19), future paths and challenges. Infection Control \& Hospital Epidemiology, 43: 1288-1290, https://doi.org/10.1017/ice.2021.226 chronology becomes particularly useful in such cases, especially because COVID-19 symptom persistence, known as long COVID-19 or postacute COVID-19 syndrome, ${ }^{6}$ is a common but separate entity concerning COVID-19 recurrence.

To evaluate the clinical characteristics of reinfection, positive recurrence, and symptom relapse, we reviewed 61 reported cases of COVID-19 recurrence in nonimmunodeficient patients indexed in MEDLINE, EMBASE, Web of Science, and LitCovid before December 3, 2020. Among the 3 current categories of recurrence, we found 18 cases of isolated positive recurrence, 44 cases of symptom relapse, and 9 cases of reinfection. In patients with reinfection or symptom relapse, the median patient age was 46 years (IQR, 32-67), and $41.7 \%$ of patients were female. Also, $49 \%$ of patients presented no clinically significant comorbidity. Interestingly, $57.9 \%$ of cases reported a more clinically important second episode of disease in comparison to the first. Additionally, fever of any grade and cough of any type were the most frequent symptoms in both episodes of disease, with a notable decrease of headaches in the second episode. For further data, refer to the Supplementary Materials (online).

The frequency of reinfection is relatively unknown, possibly due to the challenges associated with obtaining viral genome sequencing in both episodes of disease. However, there is no doubt about the possibility of reinfection by distinct SARS-CoV-2 genetic variants. ${ }^{5,8}$ Detailed investigation is still needed to determine whether there is a consistent difference between the severity of reinfection compared to the original episode and how viral-host interactions with different variants alter reinfection dynamics. To answer these questions, institutions must either increase the number of sequenced samples or develop the means to preserve samples for posterior sequencing in case of suspected reinfection.

With regard to positive recurrence, the literature shows that several factors may be relevant to the risk of positive recurrence in RT-qPCR tests: the presence of anti-RBD IgG, viral load estimated by RT-qPCR CT values, patient age, the severity of the first episode, and the total time of hospitalization. ${ }^{1-3}$ Although 